\title{
Interior Resonance Periodic Orbits in Photogravitational Restricted Three-body Problem
}

\author{
Nishanth Pushparaj and Ram Krishan Sharma* \\ Department of Aerospace Engineering, Karunya University, Coimbatore - 641114, \\ Tamilnadu, India \\ Email: ramkrishan@karunya.edu
}

\begin{abstract}
Interior resonance periodic orbits around the Sun in the Sun-Jupiter photogravitational restricted three-body problem using the method of Poincare surface of section are studied. The nature, stability and location of these periodic orbits with interior resonances of $2: 1,3: 2$ and $4: 3$ are generated for different values of Jacobi constant C. It is found that with the increase in the value of $\mathrm{C}$, these orbits transform to tidal lock, which is a rare case of resonance with 1:1. The period of time for these orbits is found to decrease with the increase in the Sun's radiation pressure. The oblateness of Jupiter is found to increase the period of time for these orbits marginally.
\end{abstract}

Keywords: Interior resonance, periodic orbits, Poincaré surface of section, solar radiation pressure, oblateness, tidal lock.

\section{Introduction}

The importance of the phenomena of resonance in the dynamic evolution of the solar system was studied by Roy and Ovenden [1]. They established that among the planetary and satellite systems, the occurrence of commensurability between the pairs of mean motions is more frequent than in a chance distribution. The existence of a mean motion resonance between a pair of objects can lead to a repeating geometrical configuration of the orbits which guarantees stability even if the resonance is not exact, since there is still the possibility of stable liberal motion around an equilibrium point. Therefore, it is important to have an understanding of the dynamics of resonance and to develop analytical models that precisely reflect the true nature of resonant interactions. Since the late twentieth century until today, the enormous number of researches have enriched the study of Restricted Three-Body Problem (RTBP), but the influence of the various perturbing forces has not been studied in many of such interesting problems. Two such forces are due to radiation pressure and oblateness. We wish to study the effect of these perturbing forces on the interior resonance periodic orbits.

The radiation force on a particle exerted by a radiating body generally consists of three terms, namely the radiation pressure, the Doppler shift of the incident radiation and the Poynting drag (Poynting [2]; Robertson [3]). The first two are radial and the third one acts opposite to the velocity vector. The latter two components are caused by absorption and subsequent re-emission of radiation and constitute Poynting-Robertson effect. Radzievskii [4] pointed out that these two effects are negligible and that the only significant force is due to radiation pressure. A number of researchers, including Chernikov [5], Perezhogin [6], Bhatnagar and Chawla [7], Schuerman [8], Simmons et al. [9], Roman [10], Kushvah and Ishwar [11] and Das et al. [12] carried out further studies to understand the motion of objects under the effect of solar radiation pressure in the RTBP with radiating primaries. The restricted problem when the three participating bodies are oblate spheroids was studied by Elipe and Ferrer [13] and El-Shaboury and El-Tantawy [14]. When one or two of the primaries are triaxial bodies, it was studied by El-Shaboury et al. [15], Khanna and Bhatnagar [16] and Sharma et al. [17].

Some of the illustrious work is done by Danby [18], Sharma and Subba Rao [19]; Subba Rao and Sharma [20] in the perturbed restricted three-body problem. Sharma [21, 22] included the oblateness of the more massive and small primary, respectively, in the photogravitational problem and studied the periodic solutions around the Lagrangian points. Orbital resonance in RTBP was studied by Peale [23], Greenberg [24] and Hadjidemetriou [25]. Further, Dutt and Sharma [26] studied the effect of the solar 
radiation pressure on the periodic orbits in the Sun-Mars system. Beevi and Sharma [27] considered the oblateness of the more massive primary (Saturn) and studied the periodic and quasi-periodic orbits in the Saturn-Titan system. Furthermore, the existence of liberation points and their linear stability as well as periodic orbits around these points when the more massive primary is radiating and the smaller is an oblate spheroid were studied by Abouelmagd and Sharaf [28]. Singh and Haruna [29] studied the periodic orbits around the triangular equilibrium points when the three participating bodies are oblate spheroids, under the effect of radiation of the main masses and small change in the Coriolis and centrifugal forces. Zotos [30] studied the case of planar restricted three-body problem by considering one of the two primaries as an oblate spheroid to study the escape and crash mechanism of periodic orbits.

The effect of radiation pressure of a source can be expressed by a mass reduction factor $q=1-\varepsilon$, where the radiation coefficient $\varepsilon$ is the ratio of the force $F_{p}$ which is caused by radiation to the force $F_{g}$ which results from gravitation, i.e., $\varepsilon=F_{p} / F_{g} . q$ is expressed in terms of particle radius ' $a$ ', density ' $\delta$ ' and radiation pressure efficiency ' $\chi$ ' (in CGS system) as

$$
q=1-\frac{5.6 \times 10^{-5}}{a \delta} \chi
$$

Knowing the mass and the luminosity of the radiating body, $\varepsilon$ can be found for any given radius and density. Solar radiation pressure force $F_{p}$ changes with distance by the same law of gravitational attraction force $F_{g}$ and acts opposite to it. Thus, Sun's resulting force acting on the particle is (Sharma [22]; Kalvouridis et al. [31]).

$$
F=F_{g}-F_{p}=\left(1-F_{p} / F_{g}\right) F_{g}=(q) F_{g}
$$

Population of asteroids in the Jovian first-order mean motion resonances 2:1, the Hecuba gap 3:2, the Hilda group 4:3 are closely linked to the orbital evolution of the giant planets. This is because of their orbital proximity to Jupiter (Brož and Vokrouhlicky [32]). In this study, we consider the more massive primary as a source of radiation and the smaller primary as an oblate spheroid. The more massive primary is the Sun and the oblate spheroid is Jupiter. The periodic orbits around the Sun with 4:3, 3:2 and 2:1 first-order interior resonances in the framework of Sun-Jupiter photogravitational restricted three-body problem (PRTBP) are studied using Poincaré surface of section (PSS) method. The study has been carried out to find the change in the period of these orbits with the effects of solar radiation pressure (SRP) and oblateness.

\section{Equations of Motion}

In the dimensionless synodic coordinate system with origin of the system positioned on the center of mass of the primaries, considering the more massive primary $(-\mu, 0)$ as a source of radiation and smaller primary $(1-\mu, 0)$ as an oblate spheroid with its equatorial plane coincident with the plane of motion, the equations of motion of the third body are (Sharma [22])

$$
\begin{gathered}
\ddot{x}-2 n \dot{y}=\frac{\partial \Omega}{\partial x} \\
\ddot{y}+2 n \dot{x}=\frac{\partial \Omega}{\partial y} \\
\Omega=\frac{n^{2}}{2}\left[(1-\mu) r_{1}^{2}+\mu r_{2}^{2}\right]+\frac{q(1-\mu)}{r_{1}}+\frac{\mu}{r_{2}}+\frac{\mu A_{2}}{2 r_{2}^{3}} \\
r_{1}^{2}=(x+\mu)^{2}+y^{2} \\
r_{2}^{2}=(x+1-\mu)^{2}+y^{2}
\end{gathered}
$$

and 


$$
\mu=m_{2} /\left(m_{1}+m_{2}\right) \leq \frac{1}{2}
$$

$m_{1}$ and $m_{2}, m_{1}>m_{2}$ are the dimensional masses of the primaries.

The mean motion $n$ of the primaries is given by

$$
n^{2}=1+\frac{3}{2} A_{2} \text { with } \mathrm{A}_{2}=\left(A E^{2}-A P^{2}\right) / 5 R^{2} ;
$$

where $\mathrm{AE}$ and $\mathrm{AP}$ are dimensional equatorial and polar radii of the smaller primary and $\mathrm{R}$ is the distance between the primaries. $r_{1}$ and $r_{2}$ are the distances from the more massive and smaller primary, respectively.

The Jacobi integral is

$$
\dot{x}^{2}+\dot{y}^{2}=2 \Omega-C
$$

\section{Poincaré Surfaces of Section}

PSS is a widely used technique in locating the periodic and quasi-periodic orbits. To determine the orbital elements of the test particles at any instant, it is necessary to know its initial position $(x, y)$ and velocity $(\dot{x}, \dot{y})$ which corresponds to a point in a four-dimensional phase space. We have constructed the PSS on the $x, \dot{x}$ plane. The initial values were selected along the $x$-axis by using intervals of length between 0.0001 and 0.01 . The magnitude of velocity vector was determined from Jacobi constant $C$. Fine discretization of positions along the x-axis guarantees an extensive coverage of the phase plane, since each trajectory regardless of the complexity of its motion has a unique path through the phase plane. By defining the plane, say $y=0$, in resulting three-dimensional space, the values of $x$ and $\dot{x}$ can be plotted every time the particle has $y=0$, whenever trajectory intersects the plane in a particular direction, say $\dot{y}>0$.

\section{Periodic Orbits around Sun in the Sun-Jupiter System}

For the Sun-Jupiter system, we have generated the PSS for the mass ratio of $\mu=0.0009537284$, taken from Sharma and Subba Rao [33], for the Jacobian constant $C=2.99$ and $q=1$ as shown in Figure 1 and for $q=0.99$ and 0.985, as shown in Figures 2 and 3, respectively. Fourth-order Runge-Kutta-Gill method is used to integrate the equations of motion (1) and (2) for generating Poincaré surface of sections. For choosing the initial conditions for numerical integration, constant values of solar radiation pressure $q$ is taken with different values of $C$. The location of periodic orbits with 4:3, 3:2 and 2:1 interior resonances (located from PSS) around the Sun are shown in Figure 4.

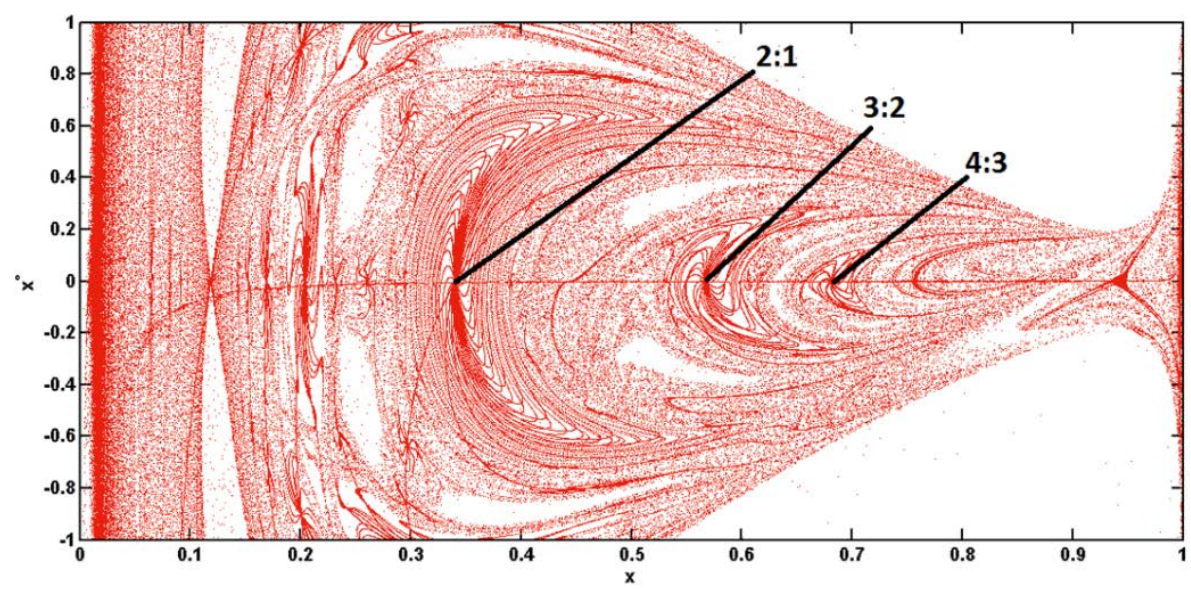

Figure 1. PSS of Sun-Jupiter system for $C=2.99$ and $q=1$. 


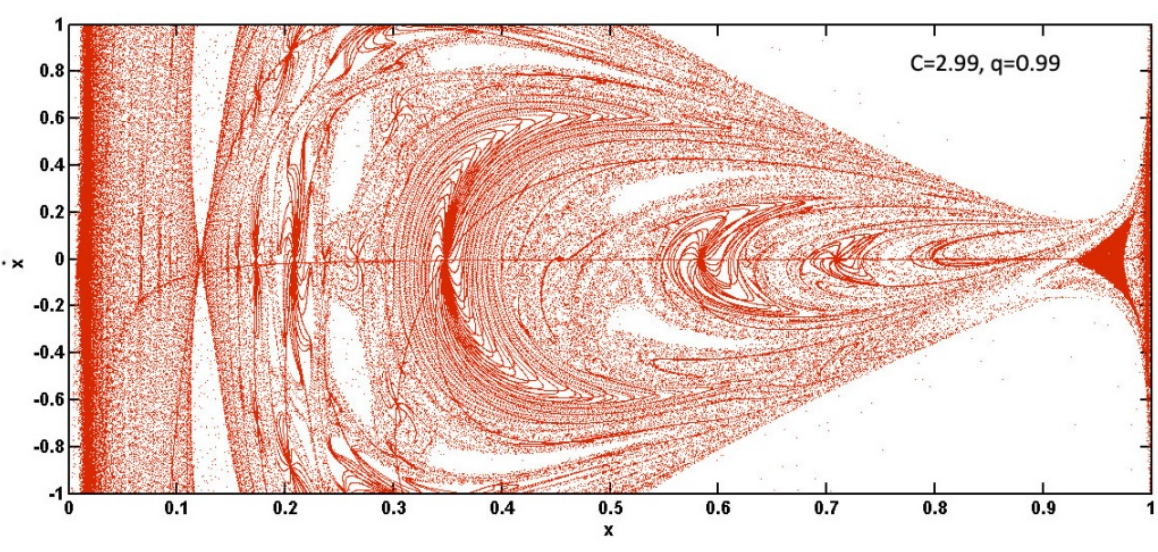

Figure 2. PSS of Sun-Jupiter system for $C=2.99$ and $q=0.99$.

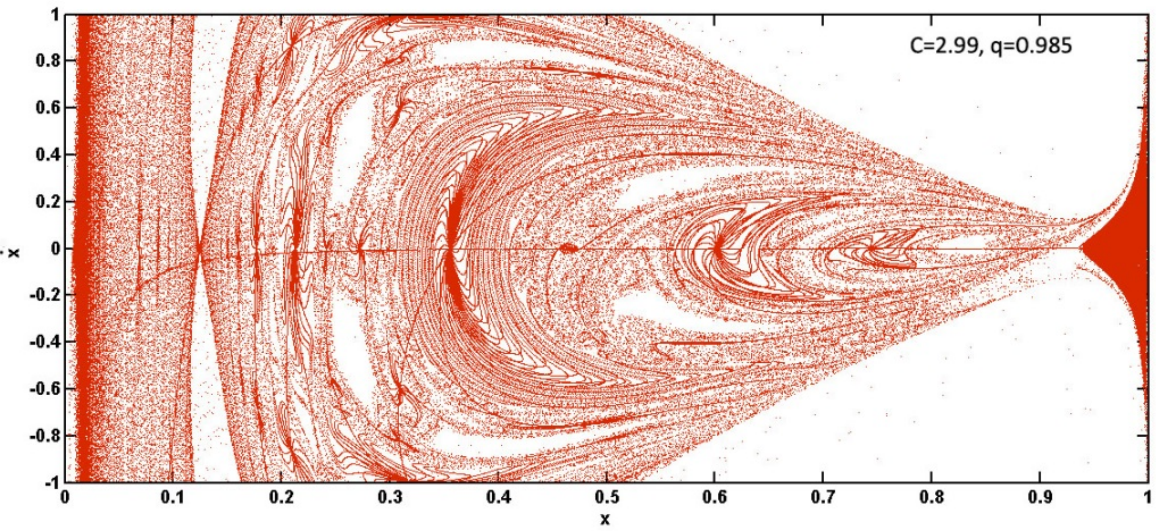

Figure 3. PSS of Sun-Jupiter system for $C=2.99$ and $q=0.985$.

\section{2:1 RESONANCE}

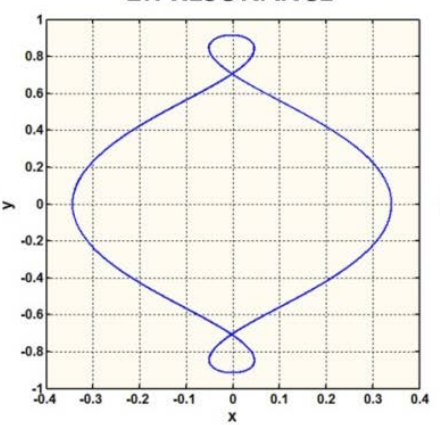

3:2 RESONANCE

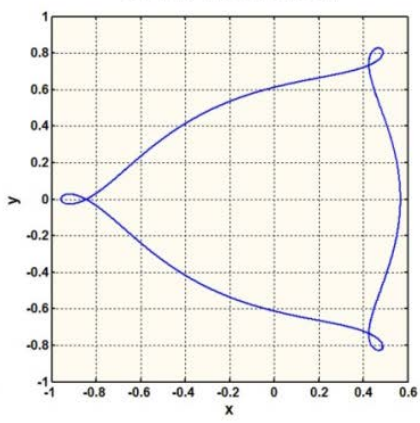

4:3 RESONANCE

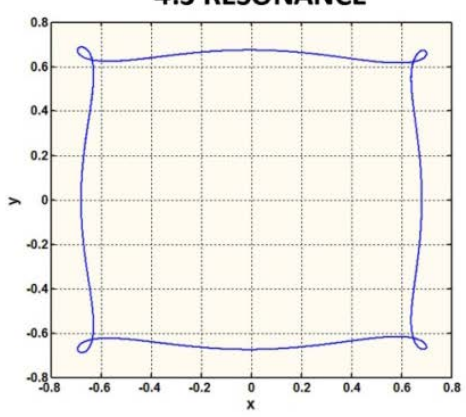

Figure 4. Periodic orbits around the Sun in Sun-Jupiter system for $C=2.99$ and $q=1$.

The periodic orbits are located from the PSS of Sun-Jupiter system and are plotted as in Figure 4, showing the interior resonance orbits around the Sun in the Sun-Jupiter system. The resonance of these periodic orbits is shown to become tidal or gravitational lock with resonance $1: 1$. The transformation of these periodic orbits with interior resonances of $2: 1,3: 2$ and 4:3 is shown in Figures 5 to 7 , respectively, by considering constant values of radiation pressure $q$ and oblateness coefficient $A_{2}$ and increasing the 
Jacobi constant $C$ from 2.99 to 3.15. Similar transformation of tidal or gravitational lock takes place when the constant values of $q$ and $A_{2}$ are different by increasing the value of $C$.

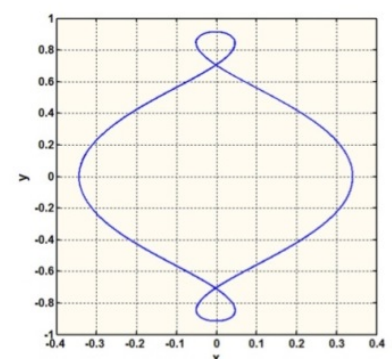

$\mathrm{C}=\mathbf{2 . 9 9}$

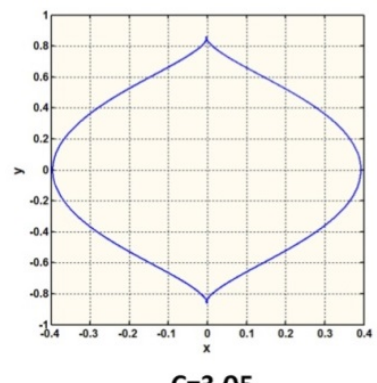

$C=3.05$

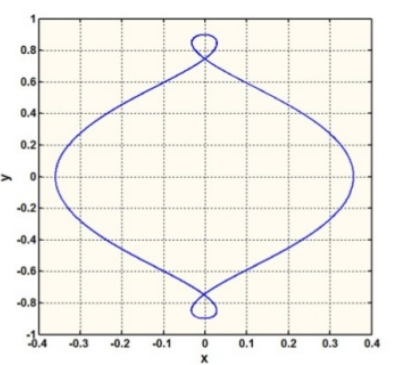

$\mathrm{C}=3.01$

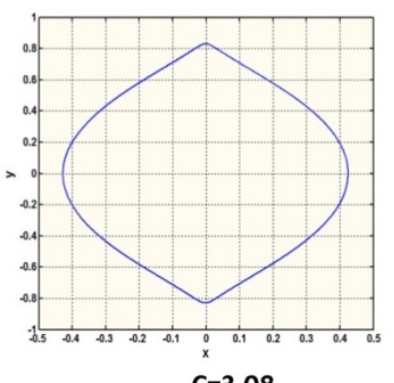

$\mathrm{C}=3.08$

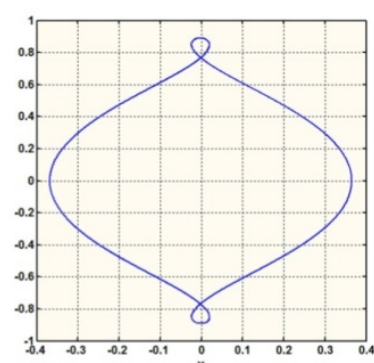

$C=3.02$

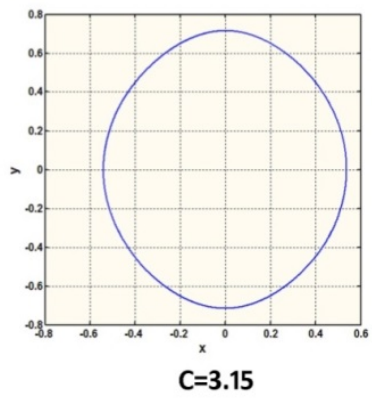

Figure 5. Transformation of 2:1 interior resonance periodic orbit with increase in Jacobi constant for $q=1$.

It is also observed that the period of time of these orbits increases with the increase in Jacobi constant $C$ for constant values of solar radiation pressure $q$ and oblateness coefficient $A_{2}$. We have discussed it in section 6 .

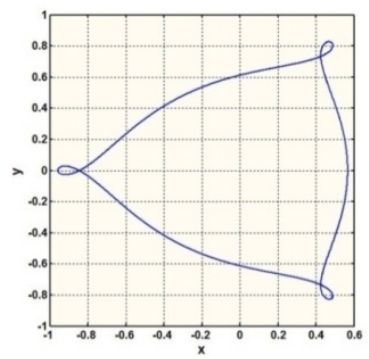

$C=2.99$

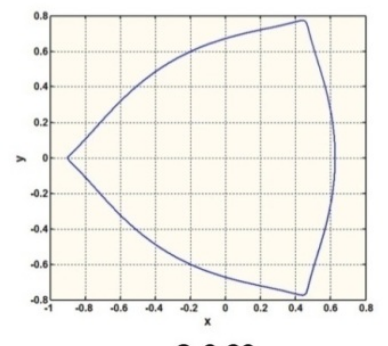

$\mathrm{C}=3.02$

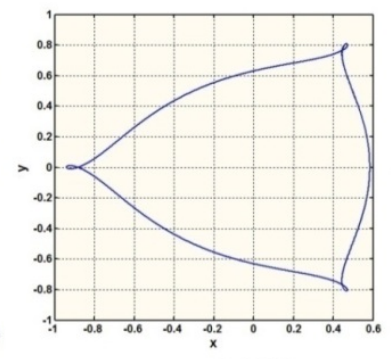

$C=3.00$

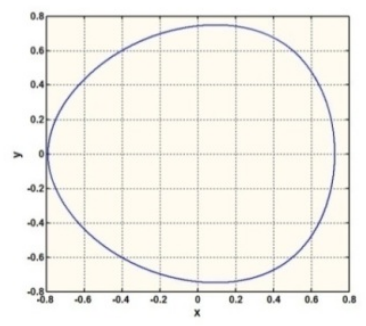

$C=3.05$

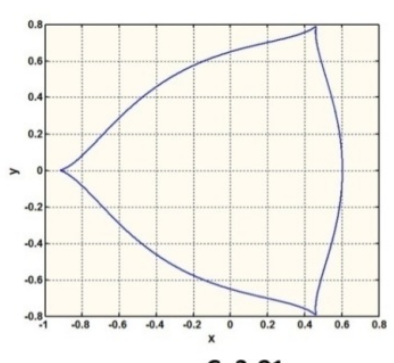

$C=3.01$

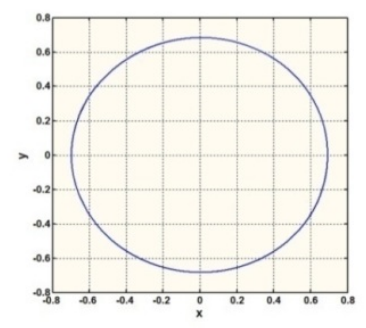

$C=3.1$

Figure 6. Transformation of 3:2 interior resonance periodic orbit with increase in Jacobi constant for $q=1$. 


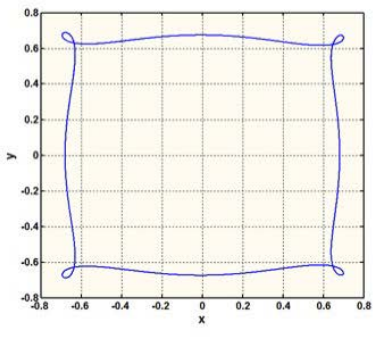

$C=2.99$

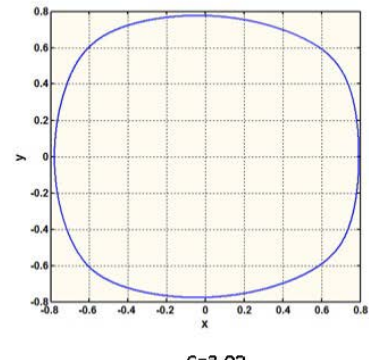

$C=3.02$
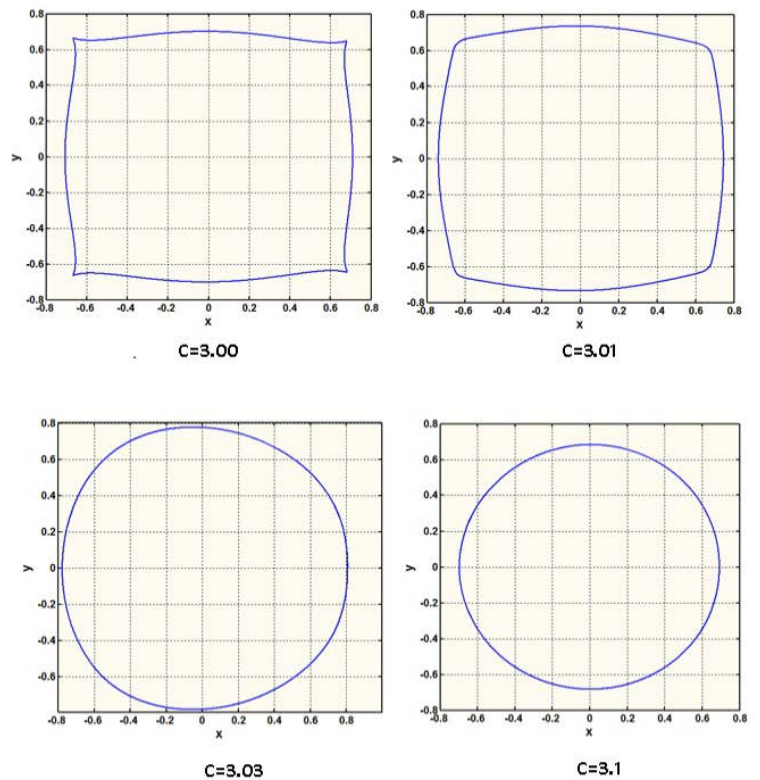

Figure 7. Transformation of 4:3 interior resonance periodic orbit with an increase in Jacobi constant when $q=1$.

\section{Stability of the Periodic Orbits}

Kolmogorov-Arnold-Moser (KAM) theory provides the stability condition for the periodic orbits in planar restricted three-body problem. PSS method is used to predict regular and chaotic behavior of a trajectory. For a regular trajectory, there exists a stable region of islands in the surfaces of section or a curve shrinking to a point and a periodic orbit exists there and a closed area around it represents quasiperiodic orbits. Any irregular distribution of points on the surfaces of section that describes the trajectory is chaotic in behavior.

In the present dynamical system considered, the KAM tori of the interior resonance orbits are used to measure the degree of stability of the periodic orbits around the Sun with respect to the region around it in the phase space. Figures 8 to 10 provide the location of the periodic orbit as a function of the Jacobi constant $C$. These results are generated from the PSS by varying the Jacobi constant $C$. In Figures 8 to 10, the solid line corresponds to the rightmost tip and the dashed line corresponds to the leftmost tip of the island obtained by PSS of the interior resonance orbits around the Sun.

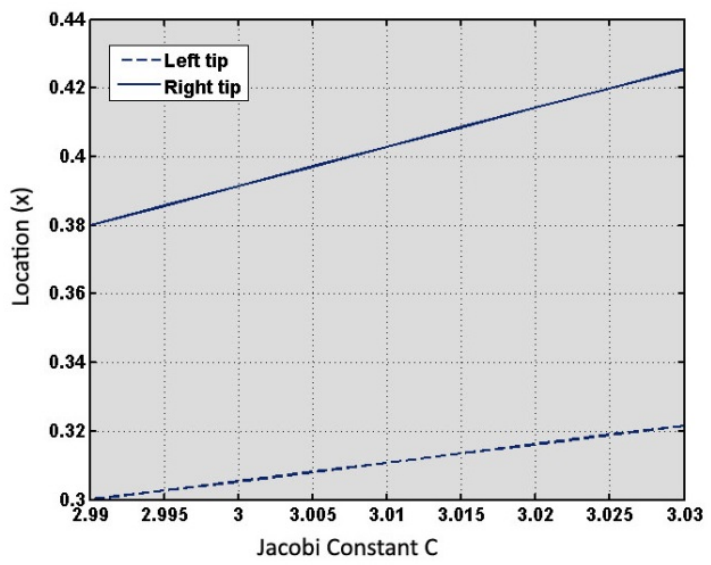

Figure 8. Width of KAM tori and location of 2:1 interior resonance orbit around Sun in Sun-Jupiter system. 


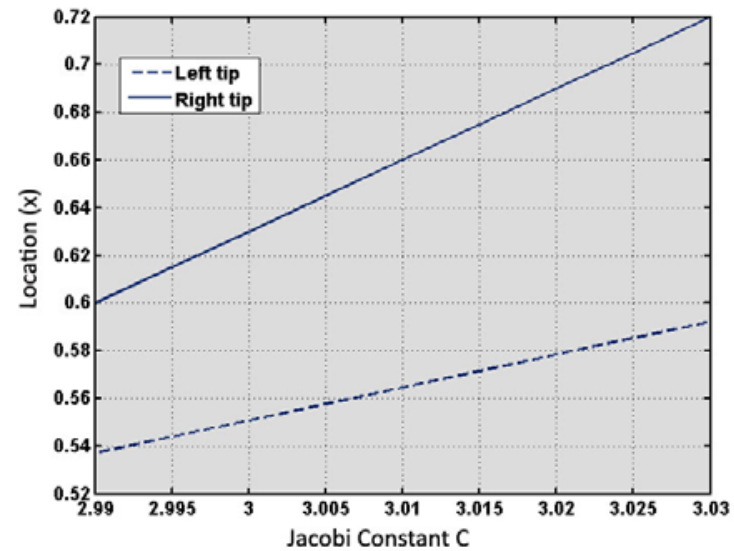

Figure 9. Width of KAM tori and location of $3: 2$ interior resonance orbits around Sun in Sun-Jupiter system.

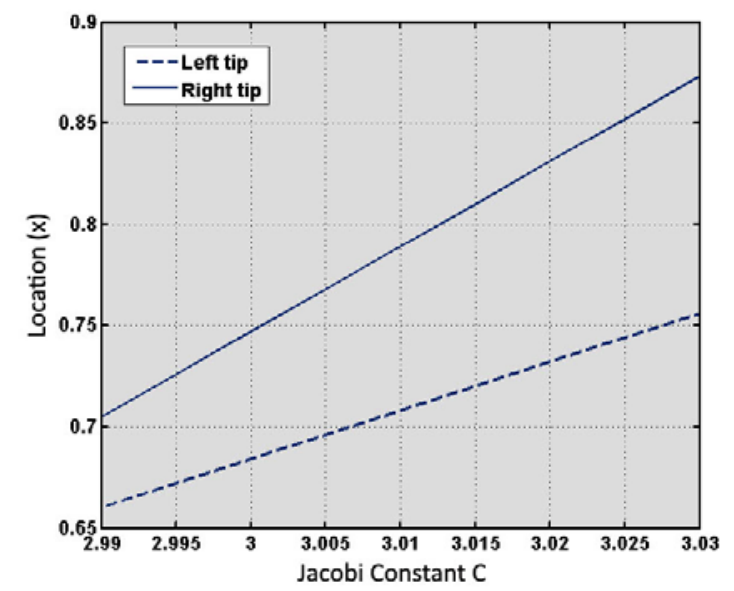

Figure 10. Width of KAM tori and the location of 4:3 interior resonance orbits around Sun in Sun-Jupiter system.

We can notice from Fig. 8 to 10, the width of KAM tori of the first-order interior resonance periodic orbits 2:1, 3:2 and 4:3 with the increase in Jacobi constant $C$. The location of these periodic orbits moves closer to the more massive primary with the increase in Jacobi constant $C$. The width of KAM tori is different for the three first-order interior resonance cases. The width of KAM tori for 2:1 resonant orbit is greater than that of 3:2 and 4:3, and it is greater for 3:2 resonant orbit than 4:3 resonant orbit.

\section{Effect of Perturbations on Period of Time of the Interior Resonance Periodic Orbits}

In the framework of the Photogravitational Restricted Three-Body Problem (PRTBP), the periodic orbits around the Sun in the Sun-Jupiter system with 2:1, 3:2 and 4:3 interior resonances with the orbit of Jupiter are studied. The solar radiation pressure plays an important role in the transformation of resonance of the periodic orbits around the Sun. It also has an effect on the period of time of these interior resonance orbits and shifts the location of these periodic orbits closer to the smaller primary. PSS has been generated for various Jacobi constant $C$ in the Sun-Jupiter system by varying the value of $q$ from 1 to 0.98 . Periodic orbits around the Sun are located from the PSS and are plotted. The variation in the period of time is noted and plotted to compare the deviation of the period of time with $q$. It shows that the period of time increases with the increase in $C$ and decreases with the increase in solar radiation pressure for all the cases of 2:1, 3:2 and 4:3 interior resonance orbits as shown in Figures 11 to 13 .

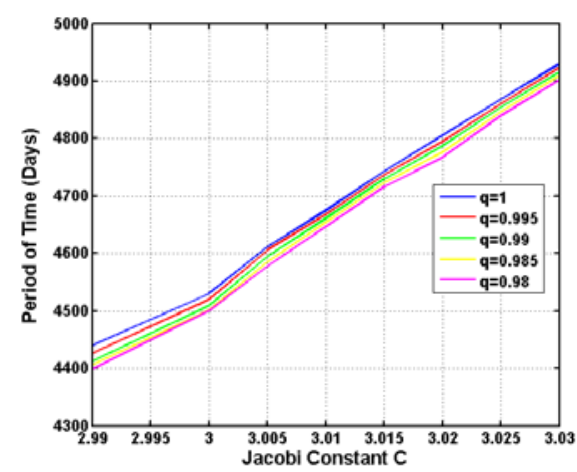

Figure 11. Period of time of $2: 1$ periodic orbits with solar radiation pressure $q$ and $C$. 


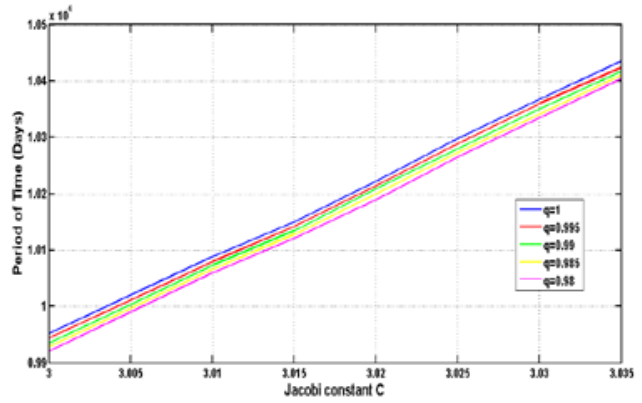

Figure 12. Period of time of $3: 2$ periodic orbits with solar radiation pressure $q$ and $C$.

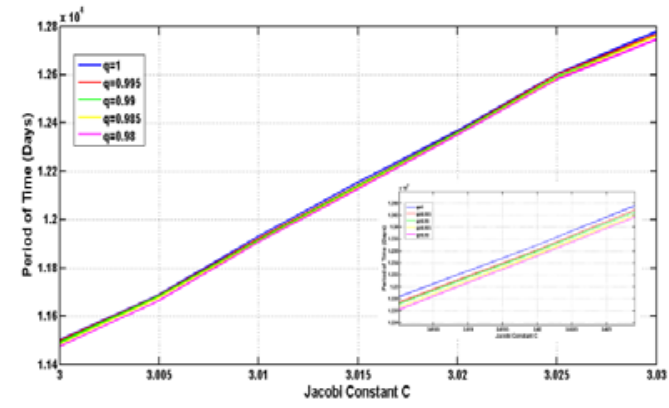

Figure 13. Period of time of $4: 3$ periodic orbits with solar radiation pressure $q$ and $C$.

We have considered the effect of oblateness $\left(A_{2}=2.117 \times 10^{-10}\right)$ of Jupiter on the motion of the particle in the PRTBP to study the deviation in the location of the periodic orbits around Sun in the Sun-Jupiter system and also the period of time deviations. It is found that there is no significant change in the location of the interior resonance periodic orbits. The time period of these orbits is found to increase marginally with the oblateness of Jupiter. The time period of interior resonance orbits is computed with and without oblateness effect. It is found that the first-order interior resonance orbits with $2: 1$ resonance have $0.146 \%$ increase in the time period. The 3:2 and 4:3 resonance orbits also have increase in the time period by $0.054 \%$ and $0.057 \%$, respectively.

\section{Summary and Conclusion}

The first-order interior resonance orbits 2:1, 3:2 and 4:3 are studied. These orbits transform into nearly circular orbits with resonance $1: 1$ or tidal lock, a rare case of resonance with 1:1, for Jacobi constant $C \geq$ 3.1. For these three type of orbits, the width of KAM tori increases with the increase in Jacobi constant. The location of these periodic orbits moves closer to the more massive primary (Sun) with the increase in Jacobi constant. The width of KAM tori of the periodic orbits 2:1 > 3:2 > 4:3. The time period of these orbits is found to increase with the increase in Jacobi constant. The time period decreases with increase in solar radiation pressure and marginally increases with oblateness of Jupiter.

\section{References}

1. A. E. Roy and M. W. Ovenden, "On the Occurrence of Commensurable Mean Motions in the Solar System," Monthly Notices of the Royal Astronomical Society, vol. 114, pp. 232-241, 1954. http://dx.doi.org/10.1093/mnras/114.2.232.

2. J. H. Poynting, "Radiation in the Solar System: Its Effect on Temperature and Its Pressure on Small Bodies," Philosophical Transactions of the Royal Society of London A, vol. 202, pp. 525-552, 1903. http://dx.doi.org/10.1098/rsta.1904.0012.

3. H. Robertson, "Dynamical effects of radiation in the solar system," Monthly Noties of the Royal Astronomical Society, vol. 97, pp. 423-437, 1937. http://dx.doi.org/10.1093/mnras/97.6.423.

4. V. V. Radzievskii, "The Restricted Problem of Three-Body Taking Account of Light Pressure," Astronomicheskii-Zhurnal, vol. 27, pp. 250-256, 1950.

5. Yu. A. Chernikov, "The photogravitational restricted three-body problem," Soviet Astronomy-AJ., vol. 14, no.1, pp. 176-181, 1970.

6. A. A. Perezhogin, "Stability of the sixth and seventh libration points in the photogravitational restricted circular three-body problem," Soviet Astronomy Letters, vol. 2, pp. 172-, 1976.

7. K. B. Bhatnagar and J. M. Chawla, "A Study of the Lagrangian Points in the Photogravitational Restricted Three-Body Problem," Indian Journal of Pure and Applied Mathematics, vol. 10, pp. 1443-1451, 1979.

8. D. W. Schuerman, "The restricted three-body problem including radiation pressure", Astrophysical Journal, vol. 238, no. 1, pp. 337-342, 1980. http://dx.doi.org/10.1086/157989. 
9. J. F. L. Simmons, A. J. C. McDonald and J. C. Brown, "The Restricted Three-Body Problem with Radiation Pressure", Celestial Mechanics, vol. 35, pp. 145-187, 1985. http://dx.doi.org/10.1007/BF01227667.

10. R. Roman, "The Restricted Three-Body Problem. Comments on the 'Spatial' Equilibrium Points", Astrophysics and Space Science, vol. 275, pp. 425-429, 2001. http://dx.doi.org/10.1023/A:1002822606921.

11. B. S. Kushvah and B. Ishwar, "Triangular equilibrium points in the Generalized Photogravitational Restricted Three Body Problem with Poynting-Robertson Drag," Review Bulletin of the Calcutta Mathematical Society, vol. 12, pp. 109-114, 2004.

12. M. K. Das, P. Narang, S. Mahajan and M. Yuasa, "Effect of Radiation on the Stability of Equilibrium Points in the Binary Stellar Systems: RW-Monocerotis, Krüger 60," Astrophysics and Space Science, vol. 314, pp. 261-, 2008. http://dx.doi.org/10.1007/s10509-008-9765-z.

13. A. Elipe and S. Ferrer, "On the equilibrium solutions in the circular planar restricted three rigid bodies problem," Celestial Mechanics, 37, 59-70, 1985. http://dx.doi.org/10.1007/BF01230341.

14. S. M. El-Shaboury and M. A. El-Tantawy, "Eulerian libration points of restricted problem of three oblate spheroids," Earth, Moon and Planets, vol. 63, pp. 23-28, 1993. http://dx.doi.org/10.1007/BF00572136.

15. S. M. El-Shaboury, M. O. Shaker, A. E. El-Dessoky, and M. A. Eltantawy, "The libration points of axisymmetric satellite in the gravitation field of two triaxial rigid bodies," Earth, Moon, and Planets, vol. 52, pp. 69-81, 1991. http://dx.doi.org/10.1007/BF00113832.

16. M. Khanna and K. B. Bhatnagar, "Existence and Stability of Libration Points in the Restricted Three Body Problem When the Smaller Primary is a Triaxial Rigid Body and the Bigger One an Oblate Spheroid," Indian Journal of Pure and Applied Mathematics, vol. 30, pp. 721-723, 1999.

17. R. K. Sharma, Z. A. Taqvi and K. B. Bhatnagar, "Existence and Stability of Libration Points in the Restricted Three-Body Problem When the Primaries Are Triaxial Rigid Bodies," Celestial Mechanics and Dynamical Astronomy, vol. 79, pp. 119-133, 2001. http://dx.doi.org/10.1023/A:1011168605411.

18. J. M. A. Danby, "Inclusion of extra forces in the problem of three bodies," Astronomical Journal, vol. 70, pp. 181-188, 1965. http://dx.doi.org/10.1086/109712.

19. R. K. Sharma and P. V. Subba Rao, "A case of commensurability induces by oblateness," Celestial Mechanics, vol. 18, pp. 185-194, 1978. http://dx.doi.org/10.1007/BF01228715.

20. P. V. Subba Rao, and R. K. Sharma, R.K. "A note on the stability of the triangular points of equilibrium in the restricted three-body problem," Astronomy and Astrophysics, vol. 43, pp. 381-383, 1975.

21. R. K. Sharma, "On Linear Stability of Triangular Libration Points of the Photogravitational Restricted ThreeBody Problem when the More Massive Primary is an Oblate Spheroid," Sun and Planetary System: Proceedings of the Sixth European Regional Meeting in Astronomy, Dubrovnik, Yugoslavia, pp. 435-436, 1982. http://dx.doi.org/10.1007/978-94-009-7846-1_114.

22. R. K. Sharma, "The linear stability of libration points of the photogravitational restricted three-body problem when the smaller primary is an oblate spheroid," Astrophysics and Space Science, vol. 135, pp. 271-281, 1987. http://dx.doi.org/10.1007/BF00641562.

23. S. J. Peale, "Orbital resonances in the solar system," Annual review of astronomy and astrophysics, vol. 14, pp. 215-246, 1976. http://dx.doi.org/10.1146/annurev.aa.14.090176.001243.

24. R. Greenberg, "Orbit - orbit resonances in the solar system: Varieties and similarities," Vistas in Astronomy, vol. 21, no. 3, pp. 209-239, 1977. http://dx.doi.org/10.1016/0083-6656(77)90031-9.

25. J. D. Hadjidemetriou, "Resonant motion in the restricted three-body problem," Celestial Mechanics and Dynamical Astronomy, vol. 56, no. 1-2, pp. 201-219, 1993. http://dx.doi.org/10.1007/BF00699733.

26. P. Dutt, and R. K. Sharma, "Evolution of periodic orbits in the Sun-Mars system," Journal of Guidance, Control and Dynamics", vol. 35, pp. 635-644, 2011. http://dx.doi.org/10.2514/1.51101.

27. A. S. Beevi and R. K. Sharma, "Oblateness effect of Saturn on periodic orbits in the Saturn-Titan restricted three-body problem," Astrophysics and Space Science, vol. 340, pp. 245-261, 2012. http://dx.doi.org/10.1007/s10509-012-1052-3.

28. E. I. Abouelmagd and M. A. Sharaf, "The motion around the libration points in the restricted three-body problem with the effect of radiation and oblateness," Astrophysics and Space Science, vol. 344, pp. 321-332, 2014. http://dx.doi.org/10.1007/s10509-012-1335-8.

29. J. Singh and S. Haruna, "Periodic Orbits around Triangular Points in the Restricted Problem of Three Oblate Bodies," American Journal of Astronomy and Astrophysics, vol. 2, pp. 22-26, 2014.

30. E. E. Zotos, "Crash test for the Copenhagen problem with oblateness," Celestial Mechanics and Dynamical Astronomy, vol. 122, pp. 75-79, 2015. http://dx.doi.org/10.1007/s10569-015-9611-x. 
31. T. Kalvouridis, M. Arribas and A.Elipe, "Parametric evolution of periodic orbits in the restricted four-body problem with radiation pressure," Planetary and Space Science, vol. 55, pp. 475-493, 2007. http://dx.doi.org/10.1016/j.pss.2006.07.005.

32. M. Brož and D. Vokrouhlicky, "Asteroid families in the first-order resonances with Jupiter," Monthly Notices of the Royal Astronomical Society, vol. 390, pp. 715-732, 2008. http://dx.doi.org/10.1111/j.1365-2966.2008.13764.x.

33. R. K. Sharma and P. V. Subba Rao, "Stationary solutions and their characteristic exponents in the restricted three-body problem when the more massive primary is an oblate spheroid," Celestial Mechanics, vol. 13, pp. 137149, 1976. http://dx.doi.org/10.1007/BF01232721 